\title{
Modelling steel-concrete interaction using the extended finite element method
}

\section{Modelación de la interacción entre acero y hormigón usando el método de los elementos finitos extendidos}

Fecha de entrega: 12 de febrero 2018

Fecha de aceptación: 14 de agosto 2018

\section{Rodrigo Gutiérrez ${ }^{1}$, Lothar Stempniewski ${ }^{1}$ and Wagner Fleming ${ }^{2}$}

${ }^{1}$ Institute of Reinforced Concrete Structures and Building Materials, Karlsruhe Institute of Technology, Kaiserstrasse 12, 76128 Karlsruhe, Germany, rodrigo.gutierrez@partner.kit.edu, lothar.stempniewski@kit.edu

${ }^{2}$ Departamento de Ingeniería Civil, Universidad Católica del Norte, Av. Angamos 0610, Antofagasta, Chile, wfleming@ucn.cl

The extended finite element method (X-FEM) is used as an alternative for the modelling of the bond behaviour between reinforcement and the surrounding concrete in reinforced concrete structures, through either reinforcing bars, fibres or others. The interface of composite materials introduces a discontinuity and, therefore, can be numerically modelled using X-FEM. This method is capable to model discontinuities without modifying the discretization by the addition of new degrees of freedom to the standard finite element approximation. Bond elements (or cohesive elements) are presented in order to be compared with the proposed alternative. The results obtained are also compared to reference solutions, showing a good agreement.

Keywords: reinforcement, finite element method, bond laws, bond elements, X-FEM, pull-out test
El método de los elementos finitos extendidos (X-FEM) se emplea como alternativa para la modelación de la unión entre el refuerzo y el hormigón circundante en estructuras de hormigón reforzado, ya sea a través de barras de refuerzo, fibras u otros. La interfaz de los materiales compuestos introduce una discontinuidad, y por lo tanto puede ser modelada numéricamente a través de X-FEM. Este método es capaz de modelar discontinuidades sin modificar la discretización mediante la adición de nuevos grados de libertad a la aproximación estándar de elementos finitos. Se presentan los elementos de unión (o cohesivos) para ser comparados con la alternativa propuesta. Los resultados se comparan también con soluciones de referencia, mostrando una buena correspondencia.

Palabras clave: refuerzo, método de los elementos finitos, leyes de unión, elementos de unión, X-FEM, ensayo de arranque

\section{Introduction}

Reinforced concrete depends on the combined action of the concrete and its embedded reinforcement to ensure proper operation during service life. This action would not be possible without a successful transfer of bond forces along the interface between both materials. In this regard, bond determines ultimately the behaviour of the structure. One of the most common and simplest ways to model this interaction is through bond laws (or cohesive laws), which belong to the group of phenomenological models. This means that for its understanding, no detailed knowledge of the underlying physical process is required. Bond constitutive laws describe relationships between the stress acting on the interface and the corresponding interfacial relative displacement (slip and opening), and define the loss of load transfer capability through the interface.
Bond laws have been incorporated into a finite element analysis using, among others, bond elements, also known as cohesive zone elements (Dugdale, 1960; Barenblatt, 1962), which are placed between bulk elements and allow the calculation of the relative displacements between concrete and the reinforcement and, therefore, the amount of bond forces along the interface.

In the present paper, as an alternative to bond elements, we extend the applications of X-FEM for the cohesive crack models (Moës and Belytschko, 2002) to the modelling of the interface between concrete and its reinforcement, through either reinforcing bars, fibres or others.

In X-FEM, in order to consider the presence of the crack, the finite element approximation is enriched with local functions based on the asymptotic and discontinuous 
features of the displacement field, thus allowing the crack to be completely independent of the mesh. This new methodology was presented in Belytschko and Black (1999) and Möes et al. (1999). A good overview of this method is given in Dolbow (1999) and Fleming (2011). Even though this method was developed to model discrete cracks, its formulation can be used to model any type of discontinuity within the material, such as those introduced by the interface. For example, Radtke et al. (2010) used a special enrichment function that can handle discrete thin fibres in a continuum matrix without meshing them.

\section{Bond constitutive laws}

The constitutive behaviour of the interface between concrete and reinforcement can be described by means of bond laws, which define relationships between the stress acting on the interface and the corresponding relative displacements. The relative displacement tangential to the interface is commonly called slip and is denoted by $s$, while the relative displacement normal to the interface is called opening and is represented by $\omega$. The bond stresses can be also divided into a tangential component $\tau$ and into a normal component $\sigma$. Usually, in the literature the tangential component $\tau$ is called bond stress, while normal component $\sigma$, radial stress; this notation will be adopted in this work.

Different kinds of bond laws can be found in the literature. Most of them can be classified into the following groups: polynomial laws, piece-wise linear laws, exponential laws and rigid-linear laws. A more detailed description can be found in van den Bosch et al. (2006). Bond laws can be also categorized in uncoupled or coupled. In an uncoupled bond law, the tangential bond stress $\tau$ is independent of the opening $\omega$, while the normal bond stress $\sigma$ is independent of the slip $s$. In a coupled bond law, the normal and tangential bond stress depend on both slip and opening.

Bond laws present generally a linear-elastic behaviour until a certain threshold that represents the bond failure point. Once this limit is exceeded, the curve starts to exhibit an irreversible softening behaviour, which is associated with a decrease of the transfer capacity of bond forces along the interface. The parameters that define the bond laws are determined empirically, and depend mostly on the geometry and properties of the materials involved, as well as on the failure mechanism. Figure 1 shows schematically a typical bond stress-slip relationship, valid for any type of reinforcement.

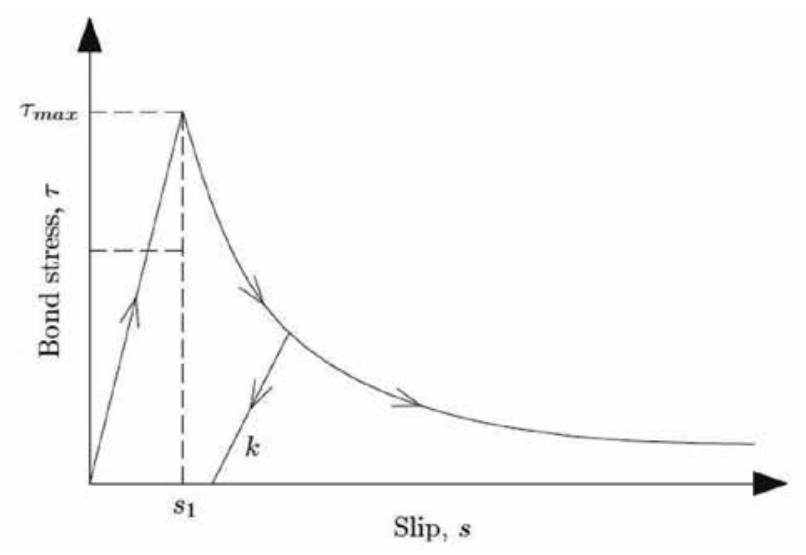

Figure 1: Bond stress-slip relationship

\section{Bond elements formulation}

Let focus our attention on Figure 2, which shows an idealization of a finite element mesh in the vicinity of the interface. The mesh is composed of traditional bulk and bond elements. Bulk elements are discretized using two-dimensional finite elements. Unlike traditional finite elements, bond elements have a width equal to zero (in Figure 2 the bond element is depicted as having a finite width in order to simplify the definitions) and no stiffness, since they do not represent a physical material, but a tool to mediate the interaction between the adjacent elements. The behaviour of bond elements is governed by bond constitutive laws, and therefore, instead of strains, the deformation field is defined in terms of the relative displacements. Bond elements contain $n$ pairs of nodes $\left(n_{i}^{+}\right.$and $n_{i}^{-}$) and each pair occupies the same location in the undeformed configuration.

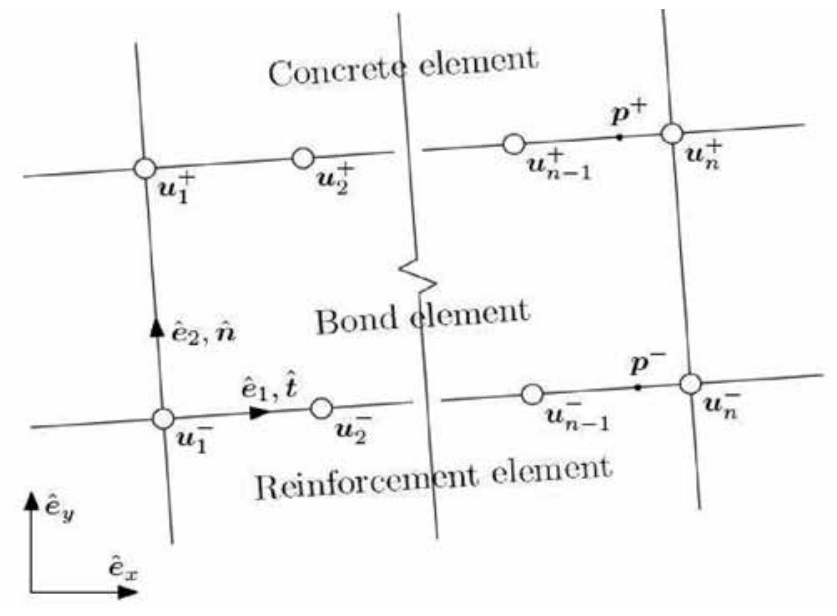

Figure 2: Finite element mesh, bond elements 
Nodes $n_{1}^{+}, \cdots, n_{n}^{+}$belong to the concrete element, while nodes $n_{1}^{-}, \cdots, n_{n}^{-}$belong to the reinforcement element. The relative displacement within the element can be determined as the difference between the displacements of any two points (point $\boldsymbol{p}^{+}(\boldsymbol{x})$ and node $\boldsymbol{p}^{-}(\boldsymbol{x})$ for example). In the global coordinate system $(x, y)$ the relative displacement is given by:

$$
\begin{aligned}
\boldsymbol{\omega}^{\text {global }}(\boldsymbol{x})= & {\left[\begin{array}{l}
\omega_{x}(x) \\
\omega_{y}(x)
\end{array}\right]=\boldsymbol{u}^{+}(x)-\boldsymbol{u}^{-}(x) } \\
& =N(x) \boldsymbol{u}_{e},
\end{aligned}
$$

where $N(x)$ is a matrix containing the shape functions and is defined as follows:

$$
\begin{aligned}
& \boldsymbol{N}(\boldsymbol{x})=\left[\begin{array}{cc}
{\left[N_{1}(\boldsymbol{x})\right.} & 0 \\
0 & N_{1}(\boldsymbol{x})
\end{array}\right] \cdots\left[\begin{array}{cc}
N_{n}(\boldsymbol{x}) & 0 \\
0 & N_{n}(\boldsymbol{x})
\end{array}\right] \\
& {\left[\begin{array}{cc}
-N_{1}(\boldsymbol{x}) & 0 \\
0 & -N_{1}(\boldsymbol{x})
\end{array}\right] \cdots\left[\begin{array}{cc}
-N_{n}(\boldsymbol{x}) & 0 \\
0 & -N_{n}(\boldsymbol{x})
\end{array}\right]}
\end{aligned}
$$

and $\boldsymbol{u}_{e}$ is a vector that groups the degrees of freedom of the bond element:

$$
\boldsymbol{u}_{e}=\left[\boldsymbol{u}_{1}^{+T} \cdots \boldsymbol{u}_{n}^{+T} \boldsymbol{u}_{1}^{-T} \cdots \boldsymbol{u}_{n}^{-T}\right]
$$

One-dimensional shape functions are use in the definition (2), which can be formulated in the natural coordinate system by the Lagrange polynomials:

$N_{i}(x(\xi))=N_{i}(\xi)=\prod_{j=1, j \neq i}^{n} \frac{\xi-\xi^{j}}{\xi^{i}-\xi^{j}}$

where $n$ represents the number of pairs of nodes of the bond element, and $\xi$ is the so called natural coordinate $(-1 \leq \xi \leq 1)$.

In the simplest case, where the bond element has only four nodes, the shape functions take the following form:

$$
\begin{aligned}
& N_{1}(\xi)=\frac{1}{2}[1-\xi], \\
& N_{2}(\xi)=\frac{1}{2}[1+\xi] .
\end{aligned}
$$

The relative displacement in the local coordinate system can be obtained by the transformation:

$$
\begin{array}{r}
\boldsymbol{\omega}(\boldsymbol{x})^{\text {local }}=\left[\begin{array}{l}
\omega_{1}(\boldsymbol{x}) \\
\omega_{2}(\boldsymbol{x})
\end{array}\right]=\left[\begin{array}{c}
\omega(\boldsymbol{x}) \\
s(\boldsymbol{x})
\end{array}\right] \\
=\boldsymbol{R}^{T} \boldsymbol{\omega}(\boldsymbol{x})^{\text {global }},
\end{array}
$$

where $\boldsymbol{R}$ is the transformation matrix from the global into the local coordinate system:

$$
R=\left[\begin{array}{ll}
\widehat{n} & \hat{\boldsymbol{t}}
\end{array}\right] .
$$

Here, $\widehat{\boldsymbol{n}}$ and $\hat{\boldsymbol{t}}$ are the unit vectors of the local coordinate system, see Figure 2.

From now on, the local relative displacement $\boldsymbol{\omega}(\boldsymbol{x})^{\text {local }}$ will be denoted only by $\boldsymbol{\omega}(\boldsymbol{x})$.

The energy balance of the system must be expanded by adding a bond (cohesive) term to the total potential energy:

$$
\delta \Pi=\delta W^{\text {int }}-\delta W^{\text {ext }}-\delta W^{\text {bond }},
$$

where $W^{\text {bond }}$ is the work of the bond stresses along the interface surface $\Omega$ and is given by:

$$
\begin{aligned}
\delta W^{\text {bond }}=\int_{\Omega+} & \delta \boldsymbol{u}^{+T} \boldsymbol{\tau}^{+} d A \\
& +\int_{\Omega-} \delta \boldsymbol{u}^{-T} \boldsymbol{\tau}^{-} d A \\
& =-\int_{\Omega} \delta\left[\boldsymbol{u}^{+}-\boldsymbol{u}^{-}\right]^{T} \boldsymbol{R}^{T} \boldsymbol{\tau} d A \\
& =-\int_{\partial \Omega} \delta \boldsymbol{\omega}^{T} \boldsymbol{R}^{T} \boldsymbol{A} \boldsymbol{\tau} d s,
\end{aligned}
$$

with:

$$
\boldsymbol{A}=\left[\begin{array}{cc}
A_{n} & 0 \\
0 & A_{t}
\end{array}\right]
$$

where $A_{n}$ and $A_{t}$ are the contact areas associated with the bond stresses $\sigma$ and $\tau$, respectively

$\tau$ is the bond stress vector and is defined by the bond constitute law, i.e. $\tau=f(\omega)$.

Replacing the discretization given in (1) in this last equation (11), an expression for the element bond force vector is obtained:

$$
\boldsymbol{F}_{e}^{\text {bond }}=-\int_{\Gamma} \boldsymbol{N}^{T} \boldsymbol{R}^{T} \boldsymbol{A} \boldsymbol{\tau}(\boldsymbol{\omega}) d \Gamma .
$$

The discretization performed above leads to a nonlinear 
problem. In this regard, to develop an efficient incremental solution procedure, it is necessary to define the element tangent matrix for the bond element. Taking the derivative of (12) with respect to the nodal displacements yields:

$$
\begin{aligned}
& \boldsymbol{K}_{e}^{\text {bond }}=\frac{\partial \boldsymbol{F}_{e}^{\text {bond }}}{\partial \boldsymbol{u}_{e}} \\
&-\int_{\Gamma} \boldsymbol{N}^{T} \boldsymbol{R}^{T} A \frac{\partial \boldsymbol{\tau}(\boldsymbol{\omega})}{\partial \boldsymbol{\omega}} \boldsymbol{R} \boldsymbol{N} d \Gamma .
\end{aligned}
$$

The evaluation of the line integrals presented above are carried out through numerical integration methods for each bond element, and the resulting element matrices are assembled in the global matrices of the system. Finally, the discrete equilibrium equation can be written as:

$$
\boldsymbol{F}^{\text {int }}=\boldsymbol{F}^{\text {ext }}+\boldsymbol{F}^{\text {bond }} \text {, }
$$

where $\boldsymbol{F}^{\text {int }}$ is the internal force vector, $\boldsymbol{F}^{\text {ext }}$ the external force vector and $\boldsymbol{F}^{\text {bond }}$ the bond force vector of the system.

\section{X-FEM formulation}

As in the case of a crack, the displacement field is discontinuous along the interface and, therefore, can be modelled using X-FEM (Figure 3).

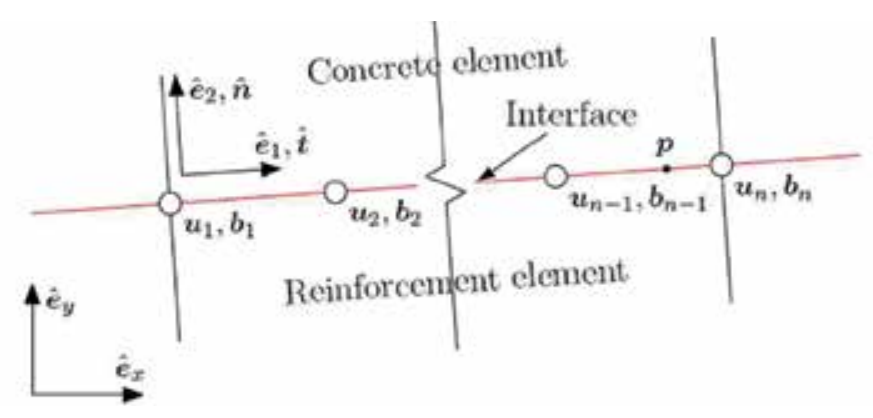

Figure 3: Finite element mesh, X-FEM

The main idea behind X-FEM is to enrich the classical finite element approximations:

$$
\boldsymbol{u}(x)=\sum_{i \in I} \boldsymbol{u}_{i} N_{i}(\boldsymbol{x}),
$$

with local information about the solution $\boldsymbol{u}$, in order to capture its local features. Here, $I$ is the set of all nodes of the system. The fact that the displacement field is discontinuous on the interface can be incorporated into the finite element approximation. The discontinuity across the interface is treated using a generalized step function, which takes the value on one side of the interface and -1 on the other, that means:

$$
H(x)=\left\{\begin{array}{rll}
1 & \text { if } x \in \Omega^{+} \\
-1 & \text { if } & x \in \Omega^{-}
\end{array}\right.
$$

Therefore, only the nodes located on the interface are enriched with this function. Figure 4 shows the enrichment strategy of the nodes.

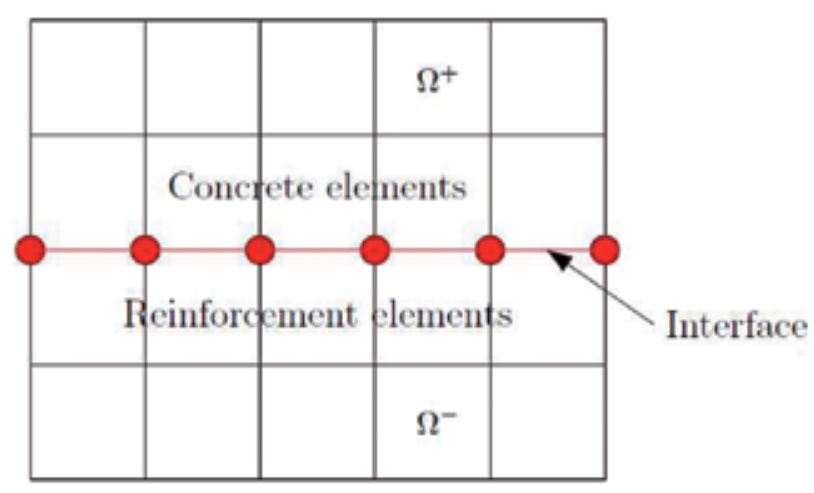

Figure 4: Enrichment strategy

Then, the X-FEM approximation of the displacement field leads to:

$$
\begin{aligned}
\boldsymbol{u}(\boldsymbol{x}) & =\sum_{i \in I} \boldsymbol{u}_{i} N_{i}(\boldsymbol{x}) \\
& +\sum_{i \in I_{H}} \boldsymbol{b}_{i}[H(\boldsymbol{x}) \\
& \left.-H\left(\boldsymbol{x}_{i}\right)\right] N_{i}(\boldsymbol{x}),
\end{aligned}
$$

where $\boldsymbol{b}_{i}$ are the extra degrees of freedom associated with the step function, and $I_{H}$ is the set of nodes enriched with this function. Special care must be taken in the evaluation of the step function of the enriched nodes, since they are located on the interface and the definition (16) is, therefore, ambiguous. In this case, the function $H\left(\boldsymbol{x}_{i}\right)$ takes the value 1 . For the calculation of the line integrals presented below it is necessary to divide the interface into segments, which are defined by the borders of the adjacent elements. For example, in Figure 3, the interface segment is limited by the nodes 1 and $n$ and groups the degrees of freedom

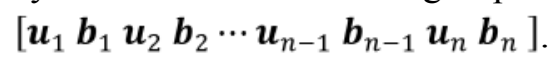

Using the same definition given in the section Bond elements formulation, the X-FEM relative displacement 
approximation in the global coordinates system can be written as:

$$
\begin{aligned}
\omega(\boldsymbol{x})^{\text {global }}= & {\left[\begin{array}{l}
\omega_{x}(\boldsymbol{x}) \\
\omega_{y}(\boldsymbol{x})
\end{array}\right]=\boldsymbol{u}(\boldsymbol{x})^{+}-\boldsymbol{u}(\boldsymbol{x})^{-} } \\
& =2 \boldsymbol{N}(\boldsymbol{x}) \boldsymbol{b}_{e}
\end{aligned}
$$

where $N(x)$ is defined as follows:

$$
\begin{gathered}
\boldsymbol{N}(\boldsymbol{x})=\left[\left[\begin{array}{cc}
N_{1}(\boldsymbol{x}) & 0 \\
0 & N_{1}(\boldsymbol{x})
\end{array}\right]\left[\begin{array}{cc}
N_{2}(\boldsymbol{x}) & 0 \\
0 & N_{2}(\boldsymbol{x})
\end{array}\right] \cdots\right. \\
{\left[\begin{array}{cc}
N_{n-1}(\boldsymbol{x}) & 0 \\
0 & N_{n-1}(\boldsymbol{x})
\end{array}\right]\left[\begin{array}{cc}
N_{n}(\boldsymbol{x}) & 0 \\
0 & N_{n}(\boldsymbol{x})
\end{array}\right]}
\end{gathered}
$$

and $\boldsymbol{b}_{\mathrm{e}}$ is a vector that groups only the degrees of freedom associated with the step function of the segment under analysis:

$$
\boldsymbol{b}_{e}=\left[\begin{array}{lllll}
\boldsymbol{b}_{1}^{T} & \boldsymbol{b}_{2}^{T} & \ldots & \boldsymbol{b}_{n-1}^{T} & \boldsymbol{b}_{n}^{T}
\end{array}\right]^{T} .
$$

The shape functions are defined in the same way as in (4).

The relative displacement in the local coordinate system can be obtained by the transformation:

$$
\begin{array}{r}
\boldsymbol{\omega}(\boldsymbol{x})^{\text {local }}=\left[\begin{array}{c}
\omega_{1}(\boldsymbol{x}) \\
\omega_{2}(\boldsymbol{x})
\end{array}\right]=\left[\begin{array}{c}
\omega(\boldsymbol{x}) \\
s(\boldsymbol{x})
\end{array}\right] \\
=\boldsymbol{R}^{T} \boldsymbol{\omega}(\boldsymbol{x})^{\text {global }},
\end{array}
$$

with $\boldsymbol{R}$ given by (8).

In this case, the element bond force vector and the element tangent matrix are written as:

$$
\begin{aligned}
& \boldsymbol{F}_{e}^{\text {bond }}=-2 \int_{\Gamma} \boldsymbol{N}^{T} \boldsymbol{R}^{T} \boldsymbol{A} \boldsymbol{\tau}(\boldsymbol{\omega}) d \Gamma, \\
& \boldsymbol{K}_{e}^{\text {bond }}=-4 \int_{\Gamma} \boldsymbol{N}^{T} \boldsymbol{R}^{T} \boldsymbol{A} \frac{\partial \boldsymbol{\tau}(\boldsymbol{\omega})}{\partial \boldsymbol{\omega}} \boldsymbol{R} \boldsymbol{N} d \Gamma,
\end{aligned}
$$

with $\boldsymbol{A}$ having the same definition as in (11).

The line integrals presented above are calculated over each interface segment, and, as in the case of the bond elements formulation, the resulting element matrices are assembled in the global matrices of the system.

\section{Numerical examples}

In order to validate the proposed alternative with regard to the bond behaviour, three pull-out tests, performed by Lettow (2011), were modelled. The test specimens correspond to a cube with $200 \mathrm{~mm}$ sides with embedded steel bars. The reinforcing bars were positioned at the centre of the specimen with embedment lengths of $3 \phi$. This length prevents the yielding of the bar before its pull-out. The diameter $\phi$ of the bars are $6 \mathrm{~mm}, 12 \mathrm{~mm}$ and $16 \mathrm{~mm}$, for the tests 1, 2 and 3, respectively. The test setup is shown in Figure 5. The models were built with 804, 768 and 770 4-nodes quadrilateral elements for the tests 1, 2 and 3, respectively. Considering that the out of plane stresses are negligible in comparison to the in plane stresses, a $2 \mathrm{D}$ plane stress model was used.

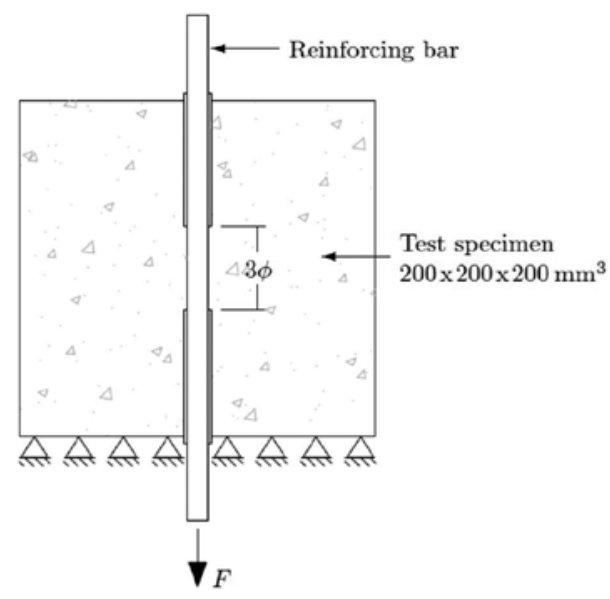

Figure 5: Test setup for the pull-out tests

Young's modulus $E$ and Poisson's ratio $v$ of the reinforcing bars are $E=200000 \mathrm{MPa}$ and $v=0.3$, while for concrete are $E=26287 \mathrm{MPa}$ and $v=0.2$. The thickness of the specimen is $h=200 \mathrm{~mm}$. In all the numerical examples, the material behaviour was assumed to be linear elastic. The equilibrium equations are solved by the NewtonRaphson method.

The bond stress-slip relationship according to Model Code 2010 (Fédération Internationale du Béton, 2012), is used:

$\tau=\left\{\begin{array}{cc}\tau_{\max }\left(\frac{s}{s_{1}}\right)^{\alpha} & \text { for } 0 \leq s \leq s_{1}, \\ \tau_{\max } & \text { for } s_{1} \leq s \leq s_{2}, \\ \tau_{\max }-\left(\tau_{\max }-\tau_{f}\right) \frac{\left(s-s_{2}\right)}{\left(s_{3}-s_{2}\right)} & \text { for } s_{2} \leq s \leq s_{3}, \\ \tau_{f} & \text { for } s_{3}<s .\end{array}\right.$

The parameters that defined the bond stress-slip relationship are the same used by Lettow (2011), and are presented in Table 1 . The normal relative displacement is assumed to be negligible. For this purpose, a penalty formulation is chosen, 
where $\sigma=\epsilon_{N} \omega$. A value of $10^{6}$ for the penalty factor $\epsilon_{N}$ is enough to avoid penetration or separation of the interface faces.

Table 1: Parameters of the bond stress-slip relationship

\begin{tabular}{|c|c|c|c|c|c|}
\hline Test & $s_{1}, \mathrm{~mm}$ & $s_{2}, \mathrm{~mm}$ & $s_{3}, \mathrm{~mm}$ & $\begin{array}{c}\tau_{\max }, \\
\mathrm{MPa}\end{array}$ & $\tau_{f}, \mathrm{MPa}$ \\
\hline 1 & 1.09 & 1.89 & 8.0 & 12.0 & 5 \\
\hline 2 & 0.77 & 1.37 & 7.5 & 11.5 & 4 \\
\hline 3 & 0.44 & 0.94 & 5.0 & 11.0 & 3 \\
\hline
\end{tabular}

* $\alpha$ was equal to 0.4 in all tests

Since the reinforcement corresponds to steel bars, the contact area $A_{t}$ associated with the tangential bond stress $\tau$ is equal to the sum of the perimeters of each bar, i.e. $n_{\phi} \pi \phi$, where $n_{\phi}$ is the number of the reinforcement rods.

Figures 6, 7 and 8 show the bond stress-slip curve for both methods exposed above. The results are in good agreement with the numerical and experimental results from Lettow (2011). On the other hand, the curves obtained by the bond elements formulation and X-FEM are identical, which shows that X-FEM can be used as an alternative for modelling of bond interaction of reinforcement concrete.

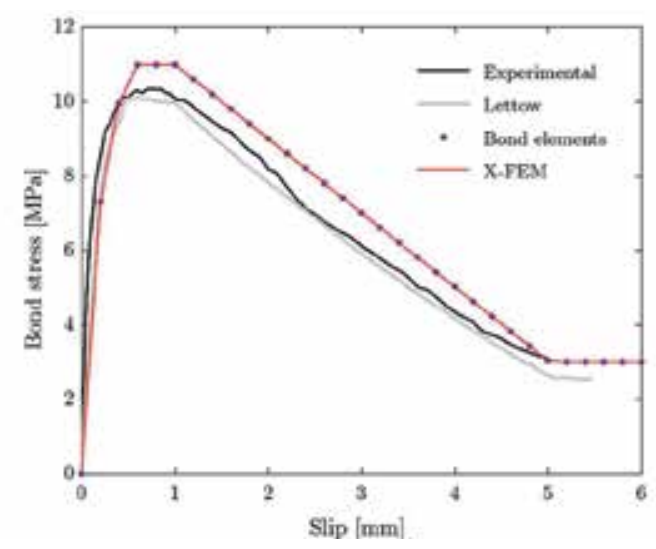

Figure 6: Bond stress-slip curve, Test 1

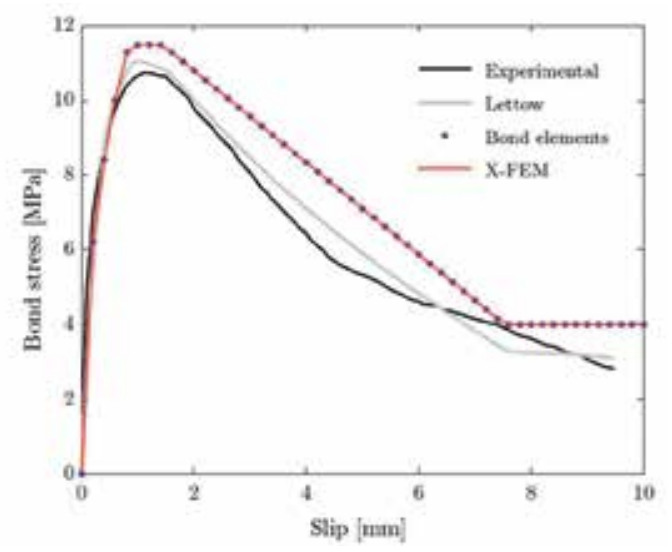

Figure 7: Bond stress-slip curve, Test 2

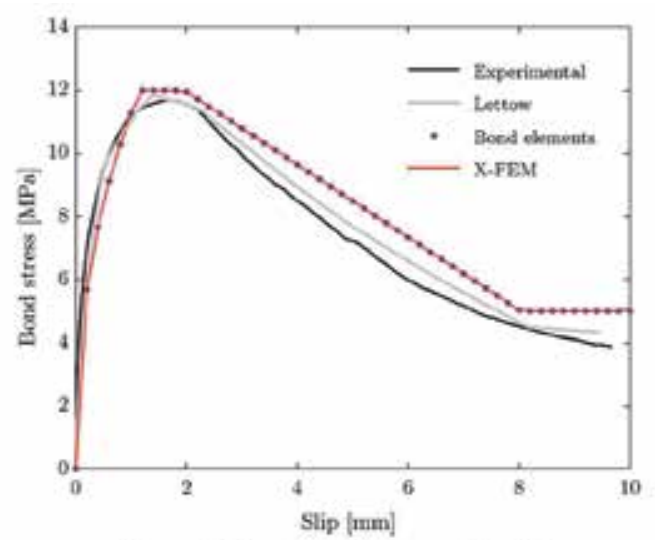

Figure 8: Bond stress-slip curve, Test 3

Figure 8: Bond stress-slip curve, Test 3

\section{Summary}

$\mathrm{X}$-FEM was used to model the bond interaction between concrete and its reinforcement, allowing, firstly, the calculation of the interfacial relative displacements and the bond stresses through bond constitutive laws, and, secondly, the transfer of bond forces between both materials. Pull-out tests were modelled considering 2D plane stress in order to validate the proposed alternative. The numerical results show good agreement with results available in literature, and are identical with those obtained by the bond elements formulation. These results confirm the reliability of the X-FEM approach as an alternative to bond elements.

The proposed methodology is particularly appropriate for the modelling of fracture in reinforced concrete structures, where the cohesive cracks are also modelled using X-FEM. In this case, the interaction between both cohesive cracks and interfaces can be effectively performed.

\section{References}

Barenblatt, G.I. (1962). The mathematical theory of equilibrium cracks in brittle fracture. Advances in Applied Mechanics 7, 55-129 Belytschko, T. and Black, T. (1999). Elastic crack growth in finite elements with minimal remeshing. International Journal for $\mathrm{Nu}$ merical Methods in Engineering 45(5), 601-620

Dolbow, J. (1999). An extended finite element method with discontinuous enrichment for applied mechanics. PhD thesis, Northwestern University, USA 
Dugdale, D.S. (1960). Yielding of steel sheets containing slits. Journal of the Mechanics and Physics of Solids 8(2), 100-104

Fédération Internationale du Béton (2012). Model Code 2010: Final draft. Switzerland: International Federation for Structural Concrete (fib): Lausanne.

Fleming, W. (2011). Quasi static and dynamic crack propagation in brittle materials with XFEM. $\mathrm{PhD}$ thesis, Universität Kassel, Germany

Lettow, S. (2011). Ein Verbundelement für nichtlineare Finite Elemente Analysen - Anwendung auf Übergreifungsstöße. $\mathrm{PhD}$ thesis, Universität Stuttgart, Germany

Moës, N. and Belytschko, T. (2002). Extended finite element method for cohesive crack growth. Engineering Fracture Mechanics 69(7), 813-833
Möes, N., Dolbow, J. and Belytschko, T. (1999). A finite element method for crack growth without remeshing. International Journal for Numerical Methods in Engineering 46(1), 131-150

Radtke, F.K.F., Simone, A. and Sluys, L.J. (2010). A partition of unity finite element method for obtaining elastic properties of continua with embedded thin fibres. International Journal for Numerical Methods in Engineering 84(6), 708-732

van den Bosch, M., Schreurs, P. and Geers, M. (2006). An improved description of the exponential $\mathrm{Xu}$ and Needleman cohesive zone law for mixed-mode decohesion. Engineering Fracture Mechanics 73(9), 1220-1234 